\title{
LEO hybrid satellite constellation design based on multi-purpose genetic algorithm to optimize cost and reliability of global coverage
}

\author{
SAEID KOHANI, PENG ZONG
}

Nanjing University of Aeronautics and Astronautics, Astronautics College, Nanjing, JiangSu, 210016, CHINA

\begin{abstract}
Assessing the reliability of systems plays an effective role in the constellation design. Genetic Algorithm can be applied for the optimization design of satellite constellation, which are imperative in various fields like communication, surveillance and navigation. Opposite goals, such as optimizing performance and reducing the number of satellites in constellations along with low cost of construction and launch, have been analyzed in this paper. In the design of constellations, launching and replacing unhealthy satellites to avoid breakdown, and the time costing has a major impact on the level of system reliability performance. A method to design hybrid constellation for communication and navigation is proposed in this paper, it takes coverage capability and precession into consideration. According to LEO constellation, The issue of optimizing the number of satellites and other effective panels in constellation design has been discussed. The genetic algorithm is designed to the hybrid LEO constellations design by using a methodology of coverage constellation. It provides the optimal solutions for enhancing capability of communication and navigation. The simulation results confirm the performance of the proposed algorithm and indicates that it is feasible and effective. Accordingly, in this paper, after designing the constellations using the genetic algorithm, we draw the final constellation diagram block and evaluating the conspicuous performance and reliability at the time of request.
\end{abstract}

Keywords: reliability, cost, satellite, LEO, constellation, genetic algorithm, coverage.

Received: January 9, 2019. Revised: February 12, 2020. Accepted: April 4, 2020. Published: April 28, 2020.

\section{Introduction}

The optimal use of the number of satellites in the constellations makes the cost less. Therefore, designers are trying to find more desirable solutions for constellation design using various numerical methods. Constellation design should also have the required reliability in different parameters. The LEO constellations, which are made up of low-altitude orbits, have many advantages for global land cover and optimal navigation. Because the low level of the LEO orbit reduces the delay in sending and receiving data and information from the satellite to the ground station or user. So, for the purpose of designing a favorable constellation of LEO, for the complete coverage of the earth, navigation, and ..., methods are used, including the genetic algorithm. Genetic Algorithm (GA) is an optimization method based on Darwin's natural and genetic selection. These algorithms are different from the traditional optimization techniques because they work with parser encoding instead of their parameters. This encoding is usually performed as a binary string, as was done in this study. The genetic algorithm optimizes and speeds up computation and accuracy of problem solving. This method has been used in several researches by Matthew L. Marcus and Daniele Mortari and William J. Mason, and ect. which optimizes the design of the orbit. Further details of this researches can be found in references [1], [2] and [3]. The LEO constellations, which are made up of low-altitude orbits, have many advantages for global land cover and optimal navigation. Because the low level of the LEO orbit reduces the delay in sending and receiving data and information from the satellite to the ground station or user. So, for the purpose of designing a favorable constellation of LEO, for the complete coverage of the earth, navigation, and ..., methods are used, including the genetic algorithm. In this study, using an orbital parameters and parameters for the formation of a satellite constellation, a genetic algorithm was developed for the design of LEO's optimal constellation. The results of using this algorithm are the formation of a LEO hybrid constellation. Considering the desirability of the LEO hybrid constellation, the results of reliability optimization and launch costs have been obtained in this study, considering the cost and reliability of the constellation performance. The global coverage of the Earth in a revisit by LEO's hybrid constellation is one of the goals of this study. Also, the desirable overlap of satellite footprints has increased the reliability and availability of satellites. Linking nearby satellites and coordinating all satellites active in hybrid constellations will require less ground stations to transmit data collected by active satellites to users. This advantage reduces the cost of designing and launching the LEO hybrid constellation.

\section{Requirements and design indicators}

The LEO constellation is a combination of uniform orbital surfaces and apart from each other which there are the same number of satellites in orbit. For LEO constellation design, Important parameters are needed for 
optimization which include $(i),(h),(F),(P)$ and $(T)$. According to global coverage theory, determined by the inclination angle the regions covered with a circular area. [4],[5], Fig. 1 is shown. If the inner radius of the cover is smaller, the extension of the coverage area is much higher. also, the gravitational angle should be equal to or greater than $3^{\circ}$, since the gravitational angle determines the outer radius of the cover circle. Because computational methods and techniques of global coverage based on the circular area of coverage are not available. in this paper, the numerical computation method has been selected which is done on the whole target grid. [6].

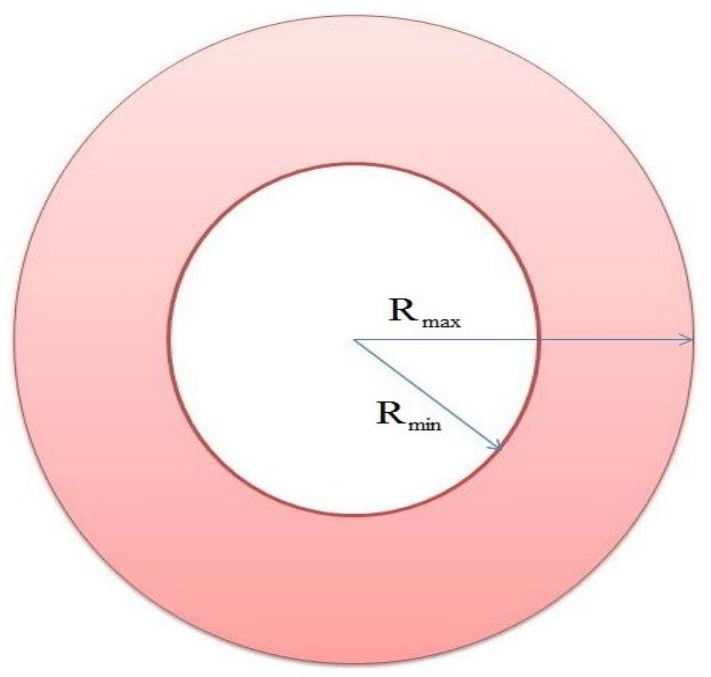

Figure. 1. The radius of coverage circle of a satellite

Observation factors of a given grid in area and time with component $\mathrm{j}$ and $\mathrm{k}$ are given by

$$
\begin{gathered}
Y_{J}=Y_{0}+Y_{1}+Y_{2}+\cdots+Y_{i=J}=\sum_{i=0}^{J} Y_{i} \\
Y_{K}=Y_{0}+Y_{1}+Y_{2}+\cdots+Y_{i=K}=\sum_{i=0}^{K} Y_{i} \\
Y_{\text {ototal }}=\sum_{i=0}^{J} \sum_{i=0}^{K} Y_{i}=\sum_{j} \sum_{k} Y_{0}(j, k)=Y_{i}(\boldsymbol{j}, \boldsymbol{k}) \\
T_{C}=\frac{Y_{\text {ototal }}}{Y_{\text {sur }}}
\end{gathered}
$$

Also, $k$ and $j$ are respectively the indicator of the target grid in time scope and the area scope, factor $Y_{\text {sur }}$ to calculate and evaluate the performance of a global satellite communications system which is based on global coverage It works by a constellation define as below

$$
\begin{aligned}
& Y_{\text {sur }}=\frac{\sum_{j} \sum_{k} Y_{0}(j, k)}{T_{c}} \\
& Y_{\text {ototal }}= \begin{cases}1 & \text { if }(m \geq 1) \\
0 & \text { otherwise }\end{cases}
\end{aligned}
$$

$\boldsymbol{Y}_{\boldsymbol{o} \text { total }}$ is the parameter is to observe each target in the area of the desired grid and mission area. also, $\boldsymbol{m}$ is the number of multi-layer global coverage in the target grid area and $\boldsymbol{T}_{\boldsymbol{c}}$ is the period of motion and return of satellites in the constellation. to optimize launch and production costs we need to minimize the number of orbital surfaces in the constellation. also, the constellation and satellite height should be at the lowest level for better performance and as the inclination angle $(\boldsymbol{i})$ is low, the area of the circular cover is increased [7]. Higher altitude and energy consumption to move to the upstream orbit and design of the geometry of the antenna will increase the cost, and therefore lower-altitude orbits have been used.

$$
\begin{gathered}
Y_{P}=\left(\frac{P}{P_{0}}\right)^{2} \\
Y_{h}=\left(\frac{h}{h_{0}}\right)^{2} \\
Y_{i}=i^{2} \rightarrow i: \text { constant for each orbital plane } \\
Y_{\text {launch }}=Y_{P}+Y_{i}+Y_{h}
\end{gathered}
$$

So the parameter of the cost of energy, production and launch are defined as follows

$$
Y_{\text {launch }}=\left(\frac{P}{P_{0}}\right)^{2}+i^{2}+\left(\frac{h}{h_{0}}\right)^{2}
$$

where $h_{0}$ and $P_{0}$ are respectively, representing the lowest satellite altitude and the number of orbit planes. To specify the configuration of the LEO constellation three special parameters $T, P$ and $F$ are required. If the time period is identical satellites in a similar geographical length and latitude, causing a collision between the satellites. This is due to the lack of precise configuration in the design of the constellation. [8],[9] For the costing reasons, It's been chosen for the lowest number $N_{\min }$ and highest number $N_{\max }$ to the number of global coverage satellites. According to the analysis of the issues mentioned, optimization design based on multi-threaded method for global coverage LEO constellation is expressed as follows

$$
\left\{\begin{aligned}
& \operatorname{Min}_{T, P, F, i, h \in S}\left\{-Y_{\text {sur }}, T, Y_{\text {launch }}, P\right\} \\
& \text { Subject to } \operatorname{Col}(T, P, F) \neq 1 \\
& F \in[0, P-1] \\
& T \in\left[N_{\text {min }}, N_{\text {max }}\right]
\end{aligned}\right.
$$


Col indicates the collision of satellites in constellations with parameters $T, P$ and $F$ also $S$ represents the search area. LEO constellation has symmetric patterns and consists of $(P)$, which represents the number of LEO orbit planes and $(T)$ representing the total number of satellites. Also, the inclination of each orbital planes with $(i)$ is expressed. So in the LEO constellation, the number of $(P)$ orbits should be uniformly distributed at angle $360^{\circ} / P$ and in each orbital plane, the angle between two adjacent satellites is equal to $360^{\circ} / \mathrm{Num}$, represents the number of satellites in each orbital plane. $T$ depends on the amount of $S$, because it shows the total number of satellites needed. Also, since the cost and number of satellites are very important in optimizing, then $T$ is a better parameter than $S$.

\section{Genetic Algorithm for hybrid LEO Constellation Design}

One of the most practical ways to solve multithreaded issues is genetic algorithm method.[10] To design a LEO constellation with genetic algorithm each of the design parameters is expressed in terms of the structure of a particular chromosome. Based on several individuals, the population size in this algorithm is determined that each individual is marked by an appropriate value, so that they meet the design requirements. As well as those who are responsible for additional requirements value is assigned a higher proportion. Therefore, the objective function must be defined on the basis of a proportional and consistent function also the objective function must also be determined to guide and control the evolutionary process. Utilizing Eq (13), taking into account the weight of different goals and their choices, the consistent and fit function is defined as follows:

$$
\begin{gathered}
F(u)_{Y_{\text {sur }}}=-w_{1} \times Y_{\text {sur }} \\
F(u)_{\mathrm{T}}=w_{2} \times T \\
F(u)_{Y_{\text {launch }}}=w_{3} \times Y_{\text {launch }} \\
F(u)_{P}=w_{4} \times P \\
F(u)_{\text {total }}=F(u)_{Y_{\text {sur }}}+F(u)_{T}+F(u)_{Y_{\text {launch }}}+F(u)_{P} \\
F(u)_{\text {total }}=-w_{1} \times Y_{\text {sur }}+w_{2} \times T \\
+w_{3} \times Y_{\text {launch }}+w_{4} \times P
\end{gathered}
$$
populations we use boundary layer matches, or binary matches. Using the constraint management method, we select the first binary set of chromosomes and from among the two chromosomes selected if both solutions are applicable in the next step their fit compatibility will be compared and will be preserved at more important classification levels. The total number of satellites is equal to the number of satellites in each orbital plane multiplied by the total number of orbital planes used.

In the equations mentioned $u$ expresses the chromosome, also $w_{1}, w_{2}, w_{3}$ and $w_{4}$ are determines the weights of 


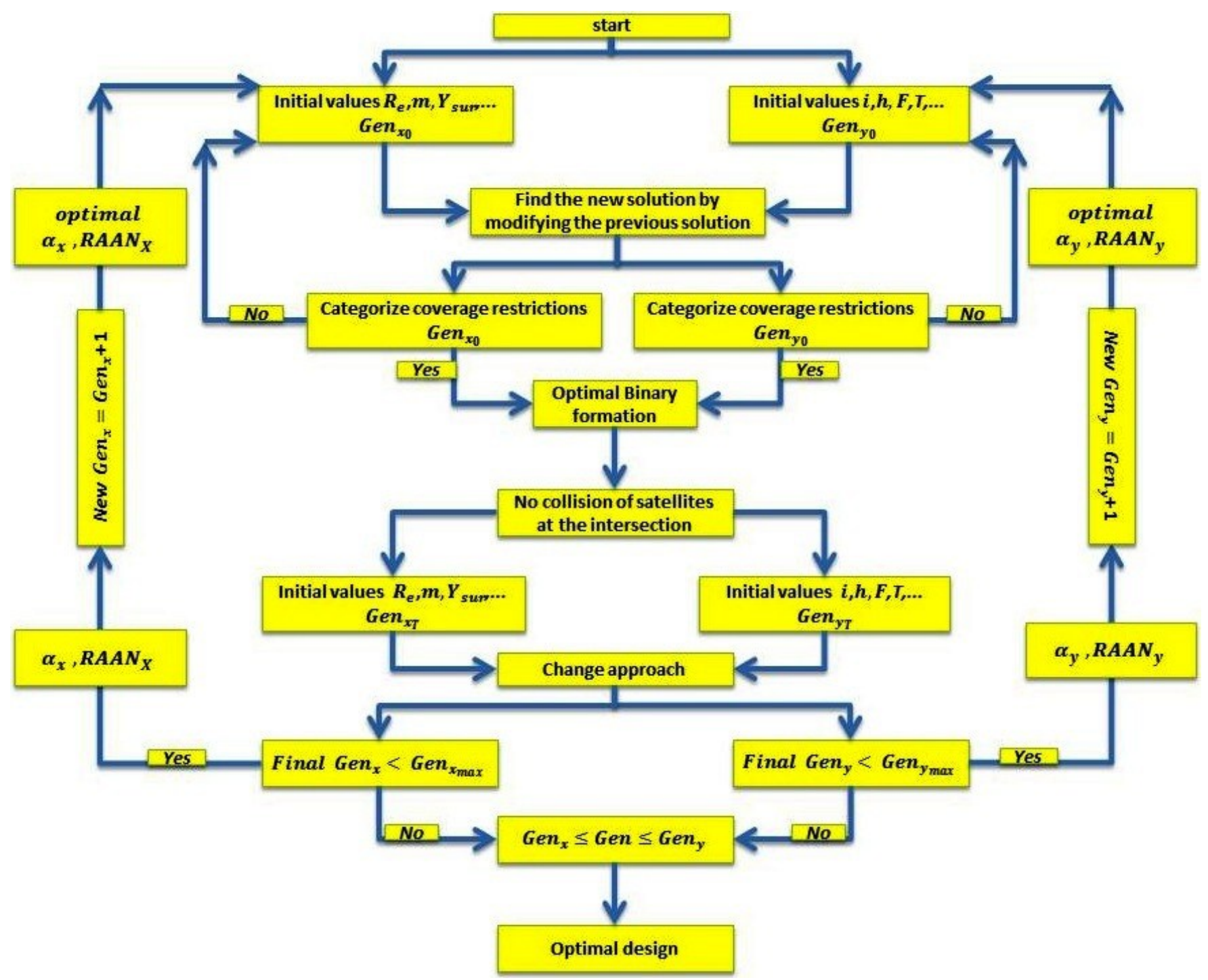

Figure. 3. Optimal Constellation Design Process Using Genetic Algorithm

Also, the type of chromosomes selection and their dependence on the number of parameters are shown in Fig. 2. So, for the design of the LEO combination constellation, optimized parameters are obtained which, in the form of chromosomal shapes, form a genetic algorithm which can be defined as follows

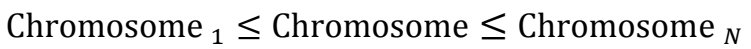

$$
N=1,2,3, \ldots \ldots, T
$$

$$
\left\{\begin{array}{c}
\text { Chromosome }_{1}=\left\{\left[P_{1}\right][N u m]\left[F_{1}\right]\left[h_{1}\right]\left[i_{1}\right][T]\right\} \\
\text { Chromosome }_{2}=\left\{\left[P_{2}\right][N u m]\left[F_{2}\right]\left[h_{2}\right]\left[i_{2}\right][T]\right\} \\
\cdot \\
\cdot \\
\cdot \\
\cdot \\
\text { Chromosome }_{T}=\left\{\left[P_{T}\right][N u m]\left[F_{T}\right]\left[h_{T}\right]\left[i_{T}\right][T]\right\}
\end{array}\right.
$$

In Eq (20), Num represents the number of satellites in each orbital plane used for each chromosome. Also, anomaly $(\alpha)$ and right ascension of ascending node (RAAN) are two important parameters in the design of the constellation LEO, which should be obtained: the optimal value of $\boldsymbol{\alpha}$ and RAAN for the distance and the rate of movement of each active satellite relative to the other ajacent satellite in the constellation, The desirability of a magnitude of $\boldsymbol{\alpha}$ and RAAN for each active satellite in the constellation makes the overlap of satellites more favorable and the probability of collision of satellites in the hybrid constellation reduces. In figure 3, The level "No collision of satellites at the intersection" after the optimal binary formation is done, then since we have two distinct sets, then collisions and intersections are not created and "change approach" is expresses the mutation and changes in genetic properties. Also shown in Fig. 3. flowchart steps proposed Genetic Algorithms which used to obtain optimal parameters for solving the LEO hybrid constellation design problem. In previous papers, the design of the genetic algorithm with 
the main parameters of the LEO constellation has not been performed. also, the number of parameter weights is low, which reduces the accuracy of algorithm calculations. In the new algorithm designed in this paper, the main parameters of the orbital and LEO constellations and polar constellations are considered. also, using the mathematical expansion in the new algorithm for the number of chromosomes selected in each gene, we can prevent the collision of satellites and by generalizing the selected genes that are derived from the orbital parameters and cost, we cover the total area of the region based on the global coverage. The population size is 840 chromosomes. In the two-point method, the children are crossover, with 12 arrays and 4200 generations needed. The fit function is also used to optimize the selection of both parent and child members. The second child inherits $80 \%$ of the genes of the parent. Also, parents are always based on the fitness function replaced. Crossover rate is $75 \%$ and Mutation rate is $0.98 \%$. In mutant operation, Using a uniform distribution of a chromosome is randomly selected and its value will change. Examples of chromosome production patterns and their results are shown in Fig. 4.

Advantages of New Genetic Algorithm In this article:

- Full global coverage.

- Multi-fold increase of coverage in the equator areas.

- Full coverage of polar regions.

- Multi-fold increase of polar regions coverage.

- Decrease of revisit time in polar and equator regions.

- Lack of satellites collide in hybrid constellation.

- Increased reliability of performance and reduce the cost of launch and system.

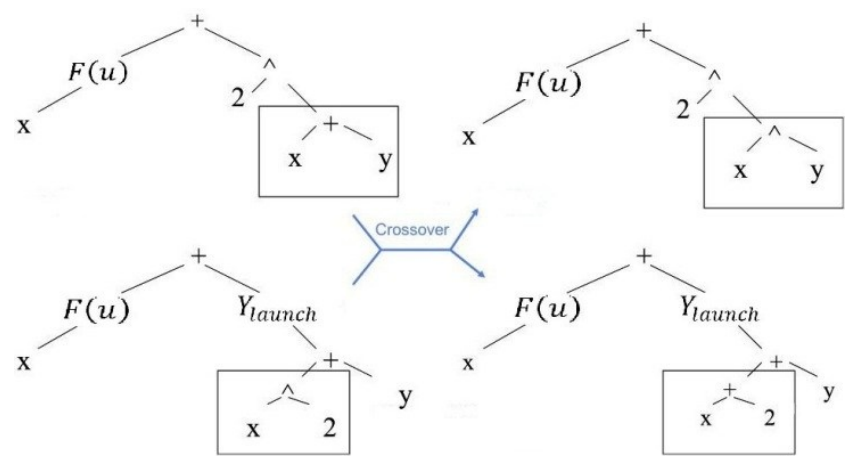

Figure. 4. Chromosome production patterns and their results

\section{Results obtained from simulation}

Considering the effects of van Allen belt radiation, The height range of the constellation LEO Hybrid is considered to be between $550 \mathrm{~km}$ and $1450 \mathrm{~km}$. Also, the inclinations of orbital planes is selected in the range of 30 degrees to 95 degrees, which changes in 5 degrees increments in each step. As a result, the two parameter groups required to form a LEO hybrid constellation are shown in Tables 1 and 2.
TABLE 1

CONSTELLATION 1

\begin{tabular}{cc}
\hline \hline Show & Value \\
\hline $\boldsymbol{i}\left({ }^{\circ}\right)$ & 80 \\
$h(\boldsymbol{k m})$ & 1400 \\
$P$ & 3 \\
$F$ & 1 \\
$T$ & 21
\end{tabular}

TABLE 2

CONSTELLATION 2

\begin{tabular}{cc}
\hline \hline Show & Value \\
\hline $\boldsymbol{i}\left(^{\circ}\right)$ & 65 \\
$h($ km $)$ & 600 \\
$P$ & 4 \\
$F$ & 0 \\
$T$ & 28
\end{tabular}

Table 1 shows that the altitude of the first constellation is $1400 \mathrm{~km}$, with 21 active satellites in that constellation, and Table 2 shows in the second constellation a height of 600 $\mathrm{km}$, with 28 active satellites. Lower altitude will increase the number of satellites, because the angle of the circle of the satellite coverage on the ground is lowered at a lower altitude. But the quality of the performance is higher and the time for sending and receiving data is less. Also, in the first constellation, due to the fact that the altitude is greater, the number of satellites is lower because the angle of the circle of the satellite coverage is higher. The orbital inclination of 80 degrees in the first constellation is due to the fact that active satellites in the constellation can cover the Arctic and Antarctic regions. The total number of active satellites in this hybrid LEO constellation is 49 satellites, which with an optimal overlap, will cover the entire earth in a revisit.

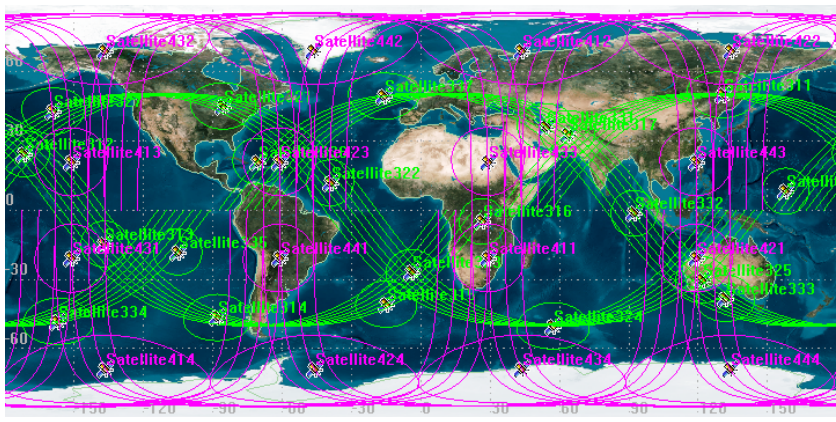

Figure 5 The Coverage of Hybrid Constellation

Figures 5 and 6 show simulations of the LEO hybrid constellation. In Figure 5, we see that the first constellation is shown in red color, due to higher altitude and 80 degree orbital inclination all areas of the Antarctic and Arctic are covered. Also, the second constellation is shown in green color which covers areas close to the equator. It is clearly 
shown in Fig. 5 that overlapping of all satellites occurs in constellations 1 and 2, This makes it an ideal global cover across the globe. In Figure 7, the effects of the use of the genetic algorithm can be seen for the number of satellites used based on the revisit time, Red color curve for designing a constellation of hybrids with a conventional method. But in the blue curve we find that the revisit time has been reduced using the new genetic algorithm in this study. Where the blue curve reaches the number of satellites ranging between 40 and 50, Can be seen that the revisit time has been optimal and desirable for the LEO hybrid constellation. In Figure 8 is shown covering power hybrid constellations. The blue curve shows that, with the increase in the number of satellites, the percentage of optimal coverage of the LEO Hybrid Constellation is further increased. Also, the purple curve shows the design of the constellation LEO with a conventional numerical method with a much lower percentage of efficiency and coverage.

\section{Initial bases of quantity and value samples}

A value sample is very necessary for the optimal design of a system, since an optimized target function needs a value sample. The process of achieving optimization is based on a comparison of volunteer samples and identification of the best sample, that is (the best choice) represents optimization. The high scores of sample values are very important because finding the best candidate or best design depends on the degree of acceptance or high scores of the value samples. All candidates with grades and their values are evaluated to determine the best assessment, this technique is used to further optimize design and production, because the design must be assessment and evaluated in order to be selected for production and construction. [11] Based on cost optimization and business applications and logical value samples, each one seeks to find the best value for optimization. An optimal design is considered for the system in question, and according to that design, the evaluation and examination of the benchmarks and indicators of net and rich net worth should be performed, this technique is used to determine pure design as a function of the features and properties of the system principle. For sample, the value of the main features of LEO constellation design, one of the main requirements of the technology of this system is considered in the optimal design, as shown in Figure 9. The attributes involved in different technologies work together to produce the gross value of system design.

\section{Modeling the constellation reliability}

For better performance and feasibility of LEO constellations, the number of $\boldsymbol{T}+\boldsymbol{i}$ satellites in the system is required. When the two satellites collide or the satellite decays, the next period of the system begins. The time to replace healthy satellites with corrupted satellites represents a period of time lost. When the
Replacement Launch $(\boldsymbol{R L})$ is started, several other failures or collisions may also occur when the number of satellites in operation is still less than $\boldsymbol{m}$. The collision or failure of the system in various modes may occur during the time period $(\boldsymbol{R} \boldsymbol{L}) . \boldsymbol{i}$ indicates the failure of a necessary satellite and the destruction of all its essential parts, that is, a satellite completely disappears.[12] During the time period $(\boldsymbol{R} \boldsymbol{L})$, the number of collisions and failures of independent satellites occurs based on a Poisson random variable.

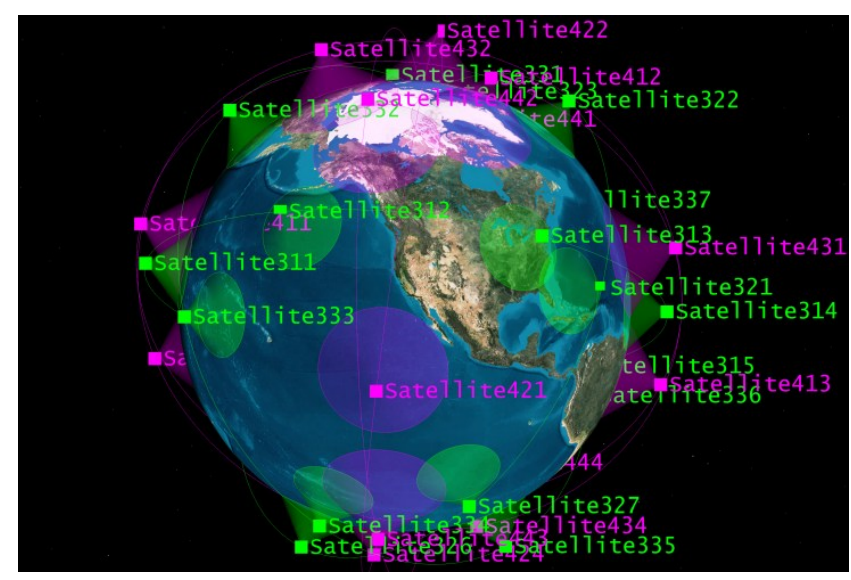

Figure 6 The Coverage of Hybrid Constellation

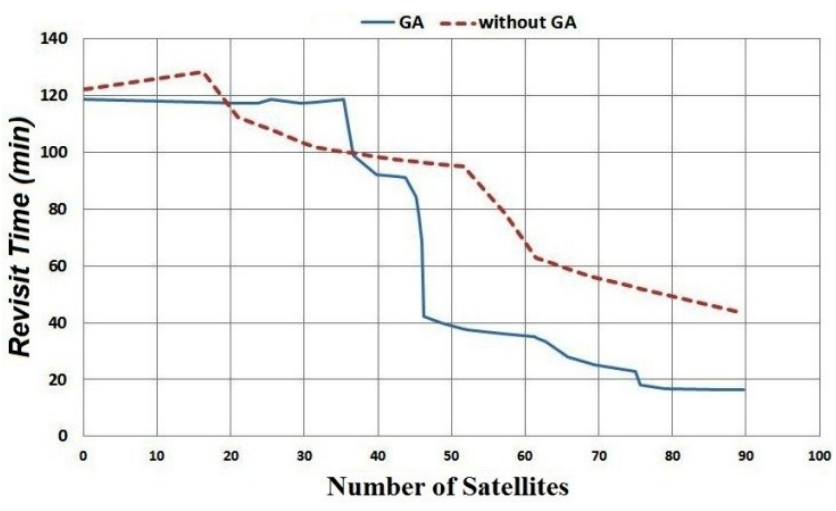

Figure 7 Hybrid LEO Constellation

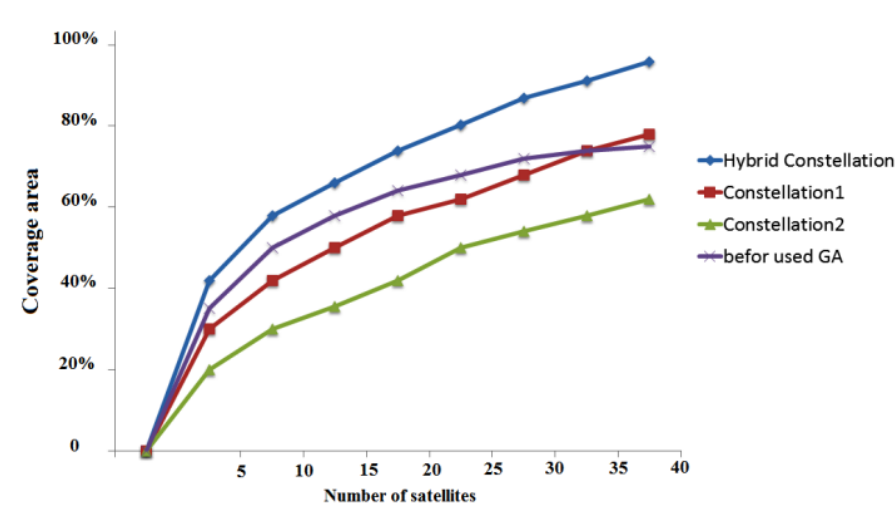

Figure 8 coverage in different constellations for global coverage of the earth 


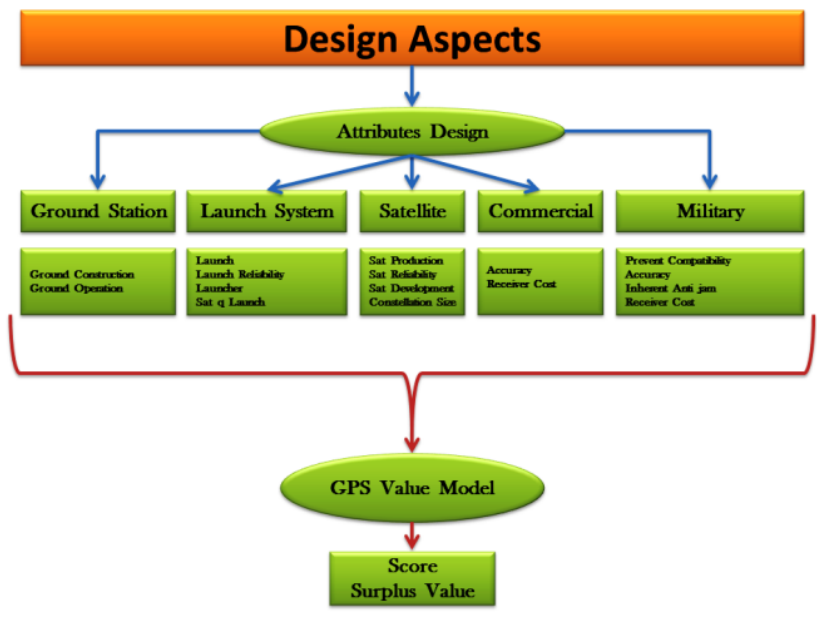

Figure 9 Sample Valuation System Properties

To reduce the error, we do not consider the collisions and failures of the satellites at the initial time interval. $P$ indicates the probability of a collision or crash is one of the satellites, which equates to the time period $(\boldsymbol{R} \boldsymbol{L})$ divided by the amount of each Satellite Reliability (SatR). [12] Mean time between failures (MTBF) and MF denotes the mean or expected wearout life (years). The reliability function of different MTBFs is illustrated in Figure.10. Also, the overall performance and reliability model cycle are shown in Figure.11. The total number of active satellites in the initial interval is given by the following formula:

$$
n+1=(P . S)+i
$$

where 1 denotes one satellite failure, the probability is

$$
p=\frac{R L}{S a t R}
$$

Given 21, by a Poisson distribution, we show the probability of a satellite breakdown over a time interval with probability $(\boldsymbol{i})$ according to the following formula,

$$
P(i) \cdot \frac{i !}{\lambda !}=e^{-\lambda}
$$

And $\frac{\lambda}{p}=n$,

Here $\lambda$ represents the average number of satellites crashed in the period of $(\boldsymbol{R} \boldsymbol{L})$. Whenever a satellite is destroyed in the constellation, a period of time from the start of a system failure occurs as an affiliate. Also, the failure cycle of the system is expressed as follows,

$$
\text { Cycle }-R L=\frac{\text { SatR }}{(P . S)+i}
$$

The average number of cycles in during a system failure period is equal to the inverse of $P(i)$. So, the probability of system failure based on the cycle is expressed as:

$$
\text { Sys Reliability }(\text { SysR })=\frac{\text { Cycle }}{P(i)}=\text { Cycle } \cdot e^{\lambda} \cdot \frac{i !}{\lambda^{i}}
$$

The total cost of the system must be minimized, the total cost of the system includes production, launch, commissioning and cost of the failure. The total cost of the system that is expected is expressed in the following formula.

$$
\mathbf{C}-\frac{S y s L}{S y s R}=\operatorname{SatC} \cdot \frac{(P . S)+i}{S a t R}
$$

$C$ represents the total cost of system failure and replacement cost, (SatC) represents the cost of the satellite, and (SatL) represents the cost of loss a system failure. In order to simplify computational operations, we consider the time unit for $\boldsymbol{C}$ and assume the reliability level $(\boldsymbol{R} \boldsymbol{L})$ and $\boldsymbol{C} \boldsymbol{y c l e}$ are similar and the number of corrupted satellites per year is equal to the number of satellites in the orbit., (P.S) $+i$ split over Sat Reliability(SatR). It is predictable that the number of failures and failures of the system in a year has a two-way relationship with Sys Reliability (SysR) of the whole system. The effects (SatR) on the amount of cost, it causes the deployment of an optimized system. So we can find the optimal number of satellites and spare parts, because the cost (SatC) and (SatR) and Sys Loss (SysL) rates have been determined. It is possible to optimize the relations in a simpler and nondimensional way by the flexibility and variations in (SatC), $(S a t R)$ to the left of the equation:

$$
\phi+(P . S)=\frac{c}{\text { SatC }} \cdot S a t R
$$

$\phi$ is normalized system cost rate.

put (25) into (26) as

$$
\phi-i=\frac{S y s L}{\text { SatC }} \cdot \frac{S a t R}{S y s R}
$$

Dimensionless ratios are expressed in the (27).

From (23),

$$
\frac{\text { Cycle }}{\text { SysR }}-\frac{R L}{\text { SatR }}=\frac{1}{(P . S)+i}
$$

The following formula, obtained from equation (21), is expressed as follows:

$$
p=\frac{\mathrm{RL}}{\mathrm{SR}}
$$




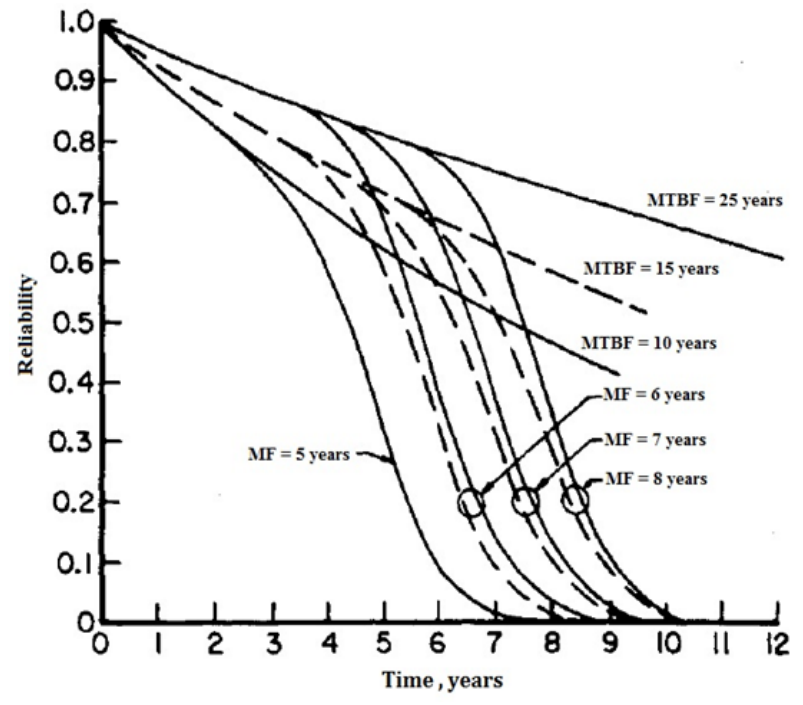

Figure 10 Satellite reliability function.

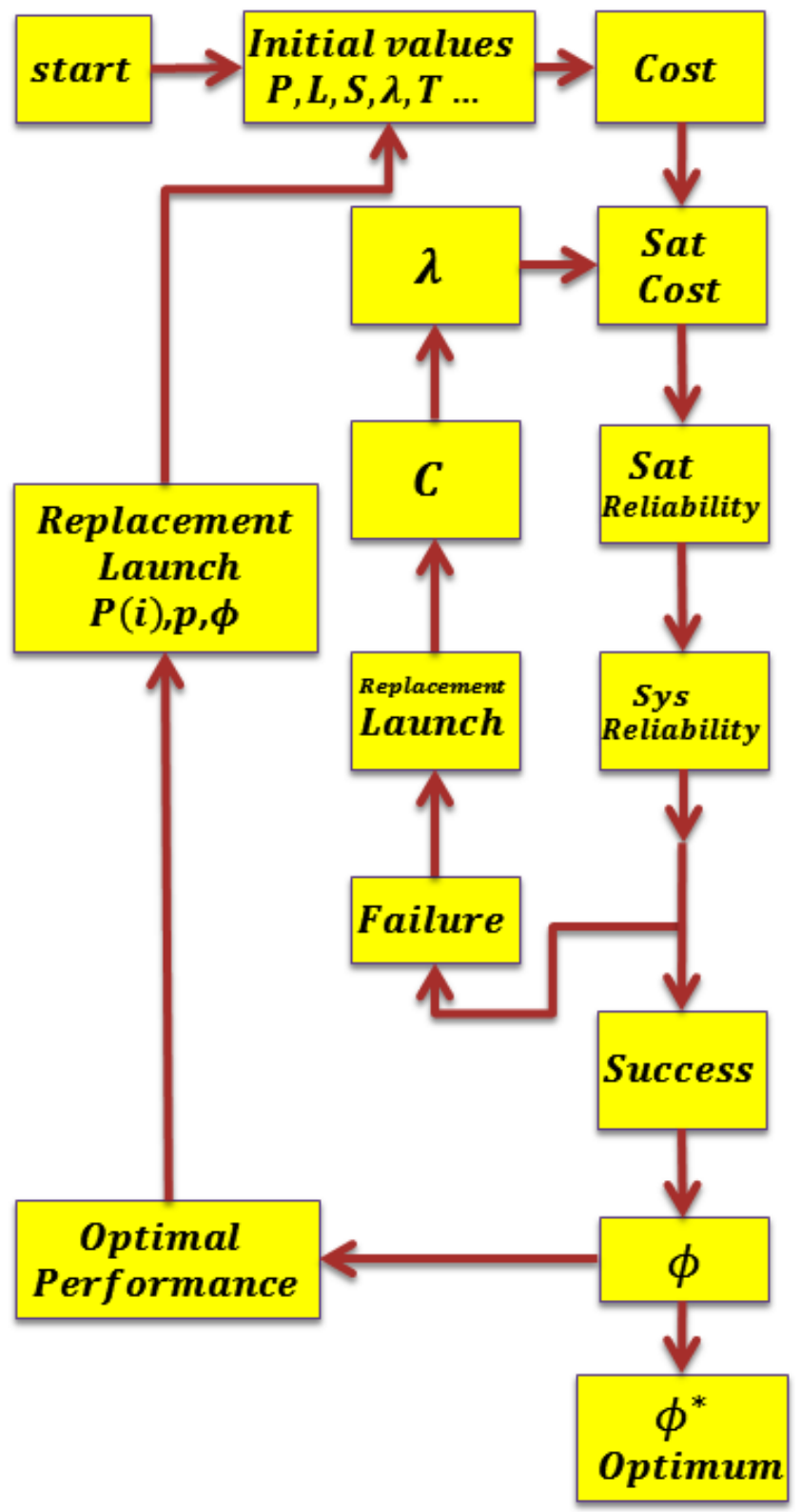

Figure 11 Reliability model cycle diagrams
This relationship represents the last fit in (27) or at least shown to be reversed in accordance with equation (24):

$$
\frac{\operatorname{SysR}}{\operatorname{SatR}} \cdot \frac{\lambda !}{i^{i}}=\left(\frac{1}{(P . S)+i}+p\right) \cdot e^{\lambda}
$$

The remainder of the optimization project is expressed as follows:

$$
L=\frac{\text { Sys } L}{\text { SatC }}
$$

The optimization result is

$$
(\min \phi)-i=\frac{L}{\left(\frac{1}{(P . S)+i}+p\right) \cdot e^{\lambda} \cdot \frac{i !}{\lambda^{i}}}
$$

Therefore, equation (32) should represent the integer, since the number of satellites is just one integer. We need to optimize the phrase in real terms and in accordance with the principles to determine the exact relationship between reliability and cost. The factorial value of (32) represents an issue because it is basically an integer. To remove this problem by using data, we use the placement of the gamma function, which is presented as follows:

$$
\Gamma(k+1)=k \text { ! }
$$

where $k$ is positive integer. So it can be used for optimization, which is expressed as follows:

$$
(\min \phi)-i=\frac{L}{\left(\frac{1}{(P . S)+i}+p\right) \cdot e^{\lambda} \cdot \frac{\Gamma(\mathrm{i}+1)}{\lambda^{i}}}
$$

Formula (34) is very complex and time-consuming. In the formula (22), the parameter $\lambda$ dependent and function of parameter $\boldsymbol{i}$. The difference between the results on the first rank is very bad and a lot. But in Formula (34), a new numerical optimization is obtained, is a Solver optimization package implemented by using an Excel spreadsheet, the across the range is

$$
\begin{gathered}
p \in[0.001,0.1] \\
L \epsilon\left[10,10^{10}\right] \\
T \epsilon[6,60] \\
S \epsilon[1,6]
\end{gathered}
$$

$S$ is number of satellites per plane, as $T / P$

In all values of $\boldsymbol{i}$, for the minimum value of $\phi$ to be expressed we use the $\phi^{*}$ sign Which makes the curve fit with a gain function. 


$$
\phi^{*} \approx f(p, L, T, S)
$$

The result is listed by

$$
\begin{aligned}
& d L=0.427 p^{2}+2.09 p+4.28 \\
& d T=d(P . S)=0.049 p^{2}+0.31+0.47 \\
& \phi^{*}=d L \cdot \log _{10} L+d T \cdot T+0.71 \\
& \phi^{*}=d L \cdot \log _{10} L+d(P . S) \cdot(P . S)+0.71
\end{aligned}
$$

TABLE 3

Compare changes after the use of GA

\begin{tabular}{ccc}
\hline \hline parameter & Without GA & With GA \\
\hline curve accuracy & $8.02 \%$ & $8.91 \%$ \\
reliability & $91.11 \%$ & $98.08 \%$ \\
cost factors & $83.74 \%$ & $78.34 \%$
\end{tabular}

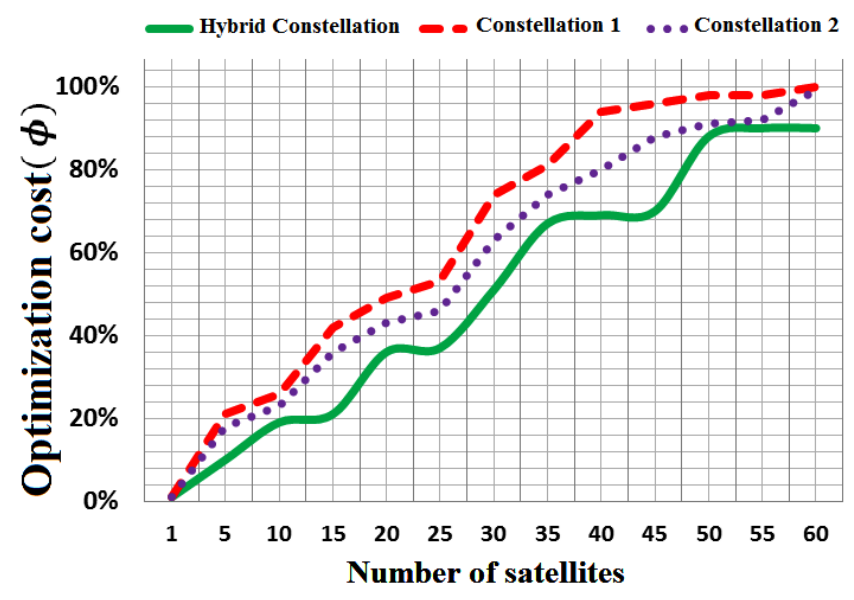

Figure.12. change the system cost

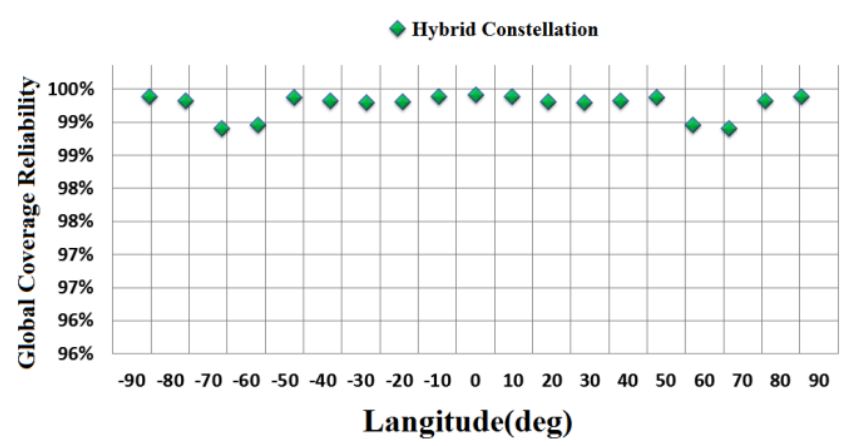

Figure.13. reliability of coverage

Numerical results are also shown in Table 3 that the cost and accuracy and the percentage of reliability are improved after using proposed Genetic Algorithm. Also, Figure. 12. Shown the change the system optimization cost level for number satellite and replacement launch. The red curve represents the costs for the formation of the first constellation and at a higher altitude, The higher the altitude is, the lower the quality, the higher the cost of launching and the lower the reliability. Also, the purple curve represents the costs of the second constellation and at lower altitudes which the costs is lower than the first constellation. Now, the green curve represents the formation of 49 satellites in the LEO Hybrid constellation, which costs much lower. Considering the advantages of the LEO Hybrid constellation, including high reliability, global coverage of the ground and access to satellites by ground stations. Figure.13. Shown that reliability of global hybrid constellation coverage on longitude. The reliability of the LEO hybrid constellation in the global coverage is between $99 \%$ and $100 \%$. Only in the geographical range between \pm 55 degrees and \pm 65 degrees, the reliability of the overall coverage is a bit lower than the other points and this is due to the presence of blind spots and also the small cavities of the coating.

\section{CONCLUSION}

The analysis of cost and reliability a hybrid LEO constellation with GA for enhancing communication and strengthening navigation is proposed in this paper. To uniquely examine all of the effective design factors, each element of the design of effective elements has been evaluated, including the probability of satellite failure, the cost of replacing bad and healthy satellites, the cost of fuel for launch, the reliability of satellite access, reliability Full coverage of the land and the possibility of satellite crashes. In order to obtain a desirable multipurpose design for the global coverage of the LEO hybrid constellation, the constellation area is also considered. The analysis of cost and reliability in a functional genetic algorithm is presented which is obtained under the influence of constraint controls. This is an applied genetic algorithm for finding optimized solutions, in which the altitudes and inclinations, functions of hybrid constellations and precession have been taken into consideration. With proposed GA, the compatibility and interoperability polar LEO constellations can be improved and effective in improving cost and reliability changes. The design of genetic algorithms to obtain the desired constellation can be used for other constellations of Mao and Geo constellations and optimize the cost and reliability of space systems. 


\section{References}

1) Optimal Earth orbiting satellite constellations via a Pareto Genetic Algorithm, William Mason, Victoria Coverstone-Carroll, John Hartmann,AIAA/AAS Astrodynamics Specialist Conference and Exhibit, $1998,10.2514 / 6.1998-4381$

2) Orbit Design for Ground Surveillance Using Genetic Algorithms, Ossama Omarabdelkhalik, Daniele Mortari, Journal of Guidance, Control, and Dynamics, 2006, Vol.29: 1231-1235, 10.2514/1.16722

3) Low Earth Orbit Debris Removal Technology Assessment Using Genetic Algorithms, Matthew L. Marcus, Raymond J. Sedwick, Journal of Spacecraft and Rockets, 2017, Vol.54: 1110-1126, 10.2514/1.A33671

4) High Order APSK Constellation Design For Next Generation Satellite Communication (AIAA 2016-5735) Farbod Kayhan, 34th AIAA International Communications Satellite Systems Conference, 2016, $10.2514 / 6.2016-5735$

5) Optimizing Flower Constellations for Global Coverage Daniel Casanova, Martin Avendano, Daniele Mortari AIAA/AAS Astrodynamics Specialist Conference, 2012, 10.2514/6.2012-4805

6) Eric Frayssinhes and Alcatel Espace. "Investigating New Satellite Constellation Geometries with Genetic lgorithms" ,AIAA-96-3636-CP

7) C-Band Multifunction Chip for the RADARSAT Constellation Mission

Martin Laflamme, Daniel Gratton, Louis Hotte, 29th AIAA International

Communications Satellite Systems Conference (ICSSC-2011), 2011, 10.2514/6.2011-8064

8) A. D. Biria, B. G. Marchand. Constellation Design for Space-Based Space Situational Awareness Applications: An Analytical Approach, Journal of Spacecraft and Rockets, March 2014, DOI: 10.2514/1.A32622.

9) Optimization of CubeSat Constellations for Uncued Electrooptical Space Object Detection and Tracking Adam C. Snow, Johnny L. Worthy III, Angela den Boer, Luke J. Alexander, Marcus J. Holzinger, David Spencer Journal of Spacecraft and Rockets, 2016, Vol.53: 401-419, 10.2514/1.A33386

10) Discretized Genetic Algorithm for satellite constellation and multiple ground antenna scheduling (AIAA 2016-2511) Hwapyong Ko, SpaceOps 2016 Conference, 2016, 10.2514/6.2016-2511

11) Dyer, J. S. (2016). Multiattribute utility theory (MAUT). International Series in Operations Research and Management Science, 233, 285-314. DOI 10.1007/978-1-4939-3094-4 8.

12) Zong Peng, Saeid Kohani. "The performance of the constellations satellites based on reliability." The Journal of Space Safety Engineering 4 (2017) pp. 112-116. 